\title{
Amphiregulin and epiregulin expression in neoplastic and inflammatory lesions in the colon
}

\author{
TAKASHI NISHIMURA, AKIRA ANDOH, OSAMU INATOMI, MAKOTO SHIOYA, \\ YUHKI YAGI, TOMOYUKI TSUJIKAWA and YOSHIHIDE FUJIYAMA
}

Department of Internal Medicine, Shiga University of Medical Science, Seta-Tukinowa, Otsu 520-2192, Japan

Received June 19, 2007; Accepted August 21, 2007

\begin{abstract}
Amphiregulin and epiregulin belong to the epidermal growth factor family and mediate the biological functions of epithelial and mesenchymal cells through epidermal growth factor receptors. In this study, we evaluated the amphiregulin and epiregulin expression in neoplastic and inflammatory lesions from the human colon. Surgicallyobtained specimens were stained using standard immunohistochemical procedures. Amphiregulin and epiregulin were not expressed in the normal colonic mucosa, but were clearly detectable in adenomas and carcinomas. Weak immunostaining was also detected in mesenchymal cells from the tumor tissues. In the active mucosa of patients with ulcerative colitis and Crohn's disease, amphiregulin was mainly expressed by the epithelial cells. In addition, positive immunostaining was also detectable in the surrounding mesenchymal cells. In conclusion, amphiregulin and epiregulin may play important roles in colonic tumor growth and mucosal repair in the inflamed mucosa of inflammatory bowel disease.
\end{abstract}

\section{Introduction}

Amphiregulin and epiregulin belong to the epidermal growth factor (EGF) family which includes EGF, transforming growth factor (TGF)- $\alpha$, heparin-binding (HB)-EGF, betacellulin and various heregulins. These factors mediate the biological functions of epithelial and mesenchymal cells through EGF receptors (EGFRs) (1). Several studies have demonstrated that the EGF family and the EGFR signalling pathway play a crucial role in the regenerative response of the mucosal damage in the gastrointestinal tract (2-4).

Amphiregulin is a 252 amino acid transmembrane glycoprotein and was originally isolated from the human breast carcinoma cell line MCF-7 (5). The mRNA expression

Correspondence to: Dr Akira Andoh, Department of Medicine, Shiga University of Medical Science, Seta-Tukinowa, Otsu 520-2192, Japan

E-mail: andoh@belle.shiga-med.ac.jp

Key words: inflammatory bowel disease, epidermal growth factor, repair for amphiregulin can be detected in a variety of carcinoma cell lines and in non-transformed epithelial and mesenchymal cells from the colon, liver, stomach, lung, breast, ovary and kidney $(6,7)$. Amphiregulin stimulates the proliferation of keratinocytes, fibroblasts and epithelial cells (8-10). In some carcinoma cell lines, amphiregulin stimulates their proliferation through autocrine mechanisms $(11,12)$. Beales demonstrated that amphiregulin stimulates the proliferation of the gastric mucosa (13).

Epiregulin is a 46 amino acid active protein and was initially purified from a conditioned medium from the fibroblast-like cell line NIH3T3/T7 (14). Epiregulin stimulates the proliferation of non-transformed fibroblasts, hepatocytes, smooth muscle cells and keratinocytes, but inhibits the growth of several tumor-derived epithelial cell lines (15-17). Lee et al reported that epiregulin null transgenic mice are highly susceptible to intestinal damage caused by the oral administration of dextran sulfate sodium (17).

These studies suggest functional roles for amphiregulin and epiregulin in neoplastic growth and/or mucosal repair, but precise studies for the in situ expression of these factors have yet to be performed in the colon. In particular, the amphiregulin and epiregulin expression in the inflamed mucosa, such as in IBD, remains unclear. In this study, we immunohistochemically evaluated the amphiregulin and epiregulin expression in neoplastic and inflammatory lesions from the human colon.

\section{Materials and methods}

Tissue samples. The diagnoses of ulcerative colitis (UC) and Crohn's disease (CD) were based on conventional clinical, endoscopic and histopathologic criteria. Surgically-obtained specimens from 5 patients with UC and 5 patients with $\mathrm{CD}$ were used after informed consent. All patients were active as defined by the colitis activity index for UC (18) and the Crohn's disease activity index (19). Surgically-resected samples of colonic adenoma $(n=5)$ and adenocarcinoma [well-differentiated $(n=3)$ and moderately-differentiated $(n=2)]$ were used. Normal colorectal tissues were obtained by surgical resection of the colon cancers at sites distant from the tumors $(n=5)$.

Immunohistochemistry. Immunohistochemical analyses were performed according to the method described in our previous 

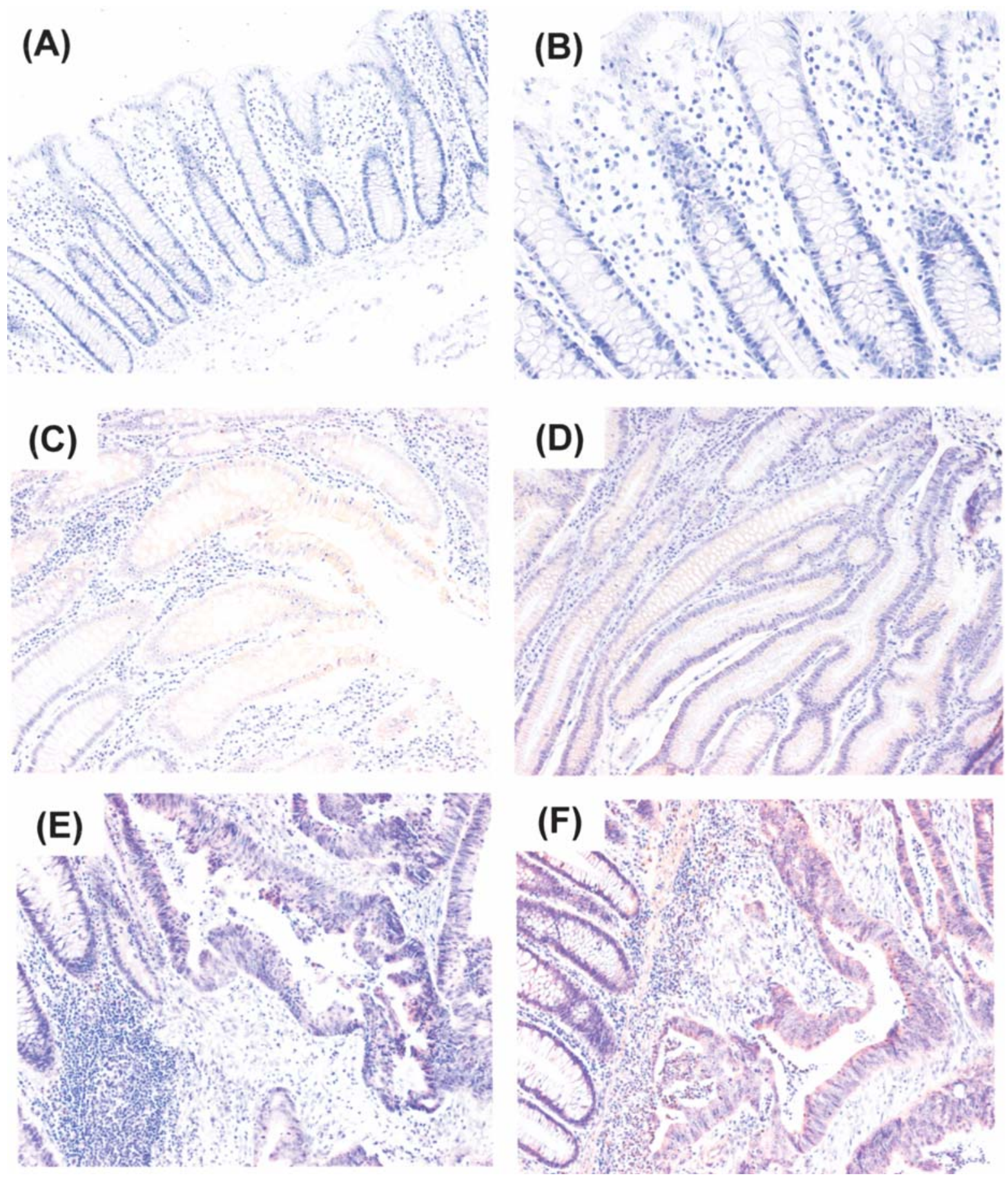

Figure 1. Amphiregulin expression in normal colonic mucosa, adenomas and carcinomas. (A) and (B), normal human colonic mucosa; (C) and (D), adenomas; and (E) and (F), carcinomas. Original magnification, except (B), x100. (B), x200.

report (20). Goat anti-human amphiregulin antibodies (R\&D Systems Inc., Minneapolis, MN) and goat anti-human epiregulin antibodies (R\&D Systems Inc.) were used as the primary antibodies. After incubation with the primary antibody, the sections were treated with a biotin-conjugated rabbit anti-goat IgG (Vector, Burlingame, CA) and avidinbiotin-peroxidase complex (ABC, Vector).

\section{Results}

Amphiregulin expression was investigated in the normal colonic mucosa, adenomas and carcinomas (Fig. 1A-F). As shown in Fig. 1A and B, amphiregulin expression was not detected in the normal colonic mucosa. However, the amphiregulin expression was clearly detected in neoplastic cells from adenomas (Fig. 1C and D) and carcinomas (Fig. 1E and F). Weak immunostaining was also detected in mesenchymal cells from the carcinoma tissues and these immunostaining patterns were detectable in all adenoma and carcinoma samples.

Epiregulin was immunostained in the adenomas and carcinomas, but the epiregulin expression was not detected in the normal colonic mucosa (Fig. 2A). Similar to the amphiregulin expression, epiregulin was detected in adenomas (Fig. 2B-D) and carcinomas (Fig. 2E and F). In the carcinoma 

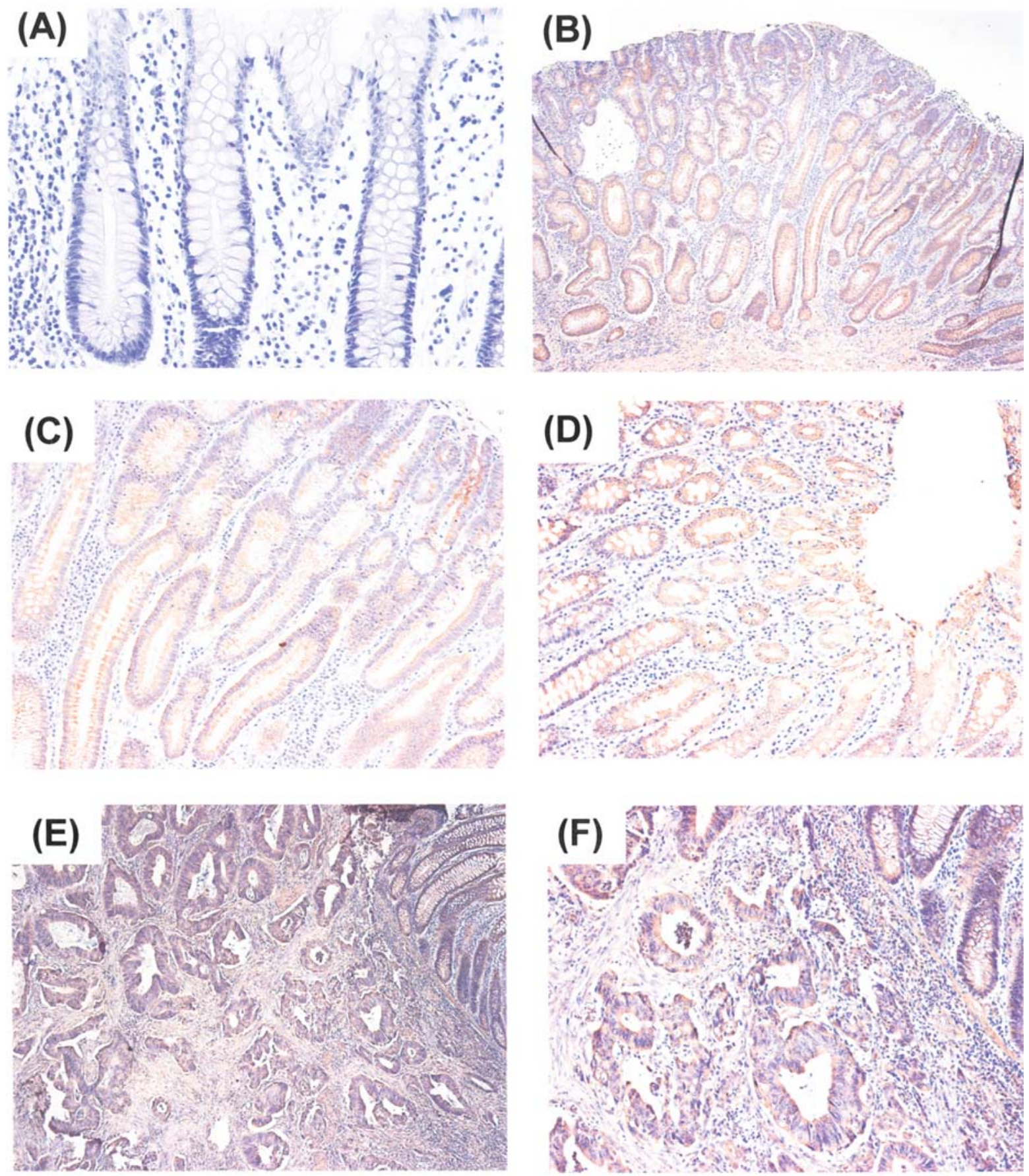

Figure 2. Epiregulin expression in normal colonic mucosa, adenomas and carcinomas. (A) Normal human colonic mucosa; (B) to (D), adenomas; and (E) and (F), carcinomas. Original magnification, except (A), x100. (A), x200

samples, the mesenchymal cells were also immunopositive for the epiregulin expression. These immunostaining patterns were detected in all adenoma and carcinoma samples.

The amphiregulin expression was evaluated in the active IBD mucosa. In the active mucosa of UC patients (Fig. 3A and B), amphiregulin was mainly expressed by the epithelial cells. In addition, positive immunostaining was detected in the mesenchymal cells. Similar immunostaining patterns were detected in the active mucosa of patients with Crohn's disease (Fig. 3C and D). The amphiregulin and epiregulin expression was similarly detectable in all samples from the UC and CD patients.
Epiregulin was immunostained in the active IBD mucosa. As with amphiregulin immunostaining, epiregulin was expressed by the epithelial cells in the active mucosa of UC (Fig. 4A and B) and CD patients (Fig. 4C and D). The mesenchymal cells were also immunopositive for epiregulin expression. Similar immunostaining patterns were observed in all samples from the IBD patients.

\section{Discussion}

This study demonstrated the amphiregulin and epiregulin expression in neoplastic lesions from the colon and in the 

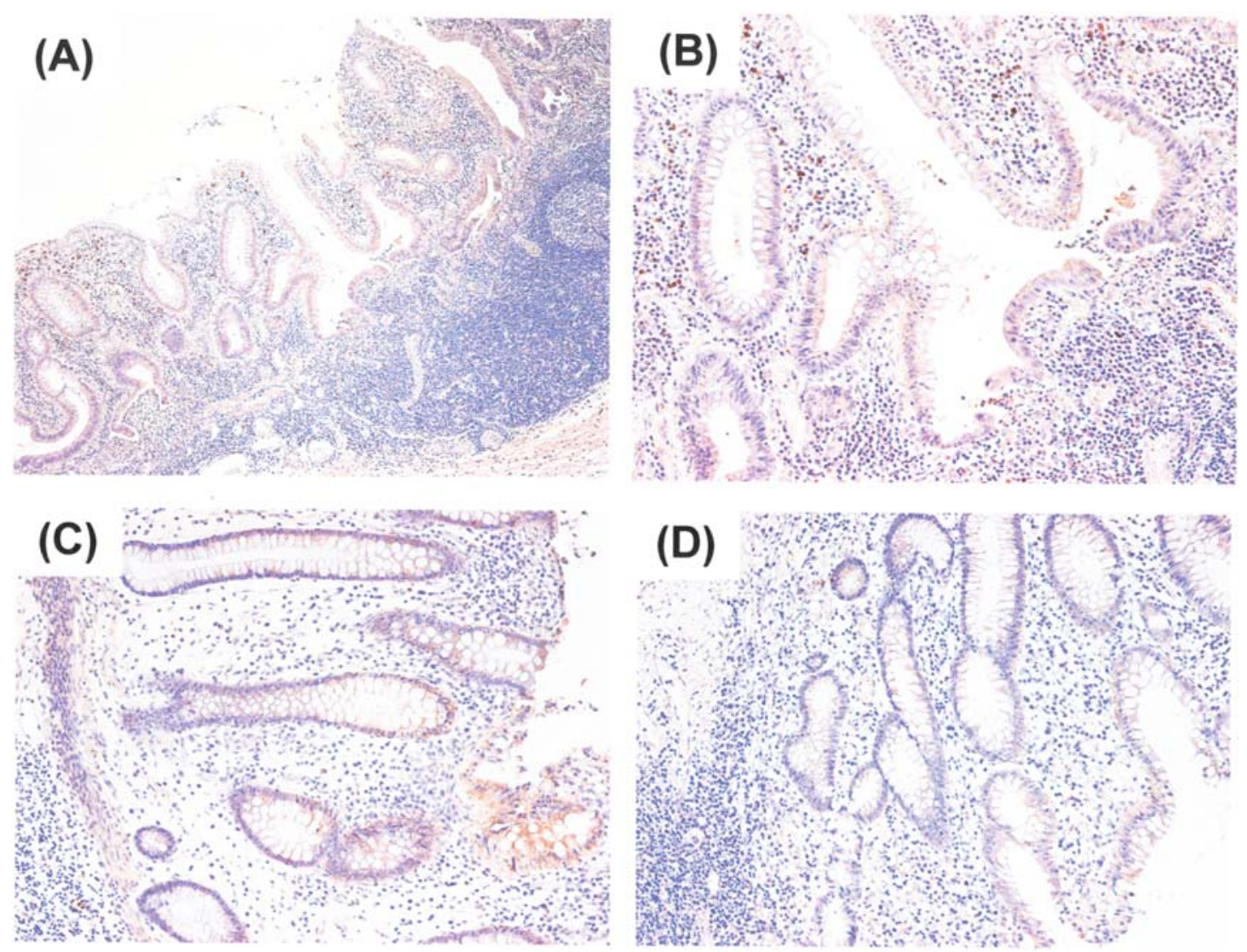

Figure 3. Amphiregulin expression in IBD mucosa. (A) and (B), ulcerative colitis; and (C) and (D), Crohn's disease. Original magnification (A) x100 and (B) to (D) $\mathrm{x} 200$.
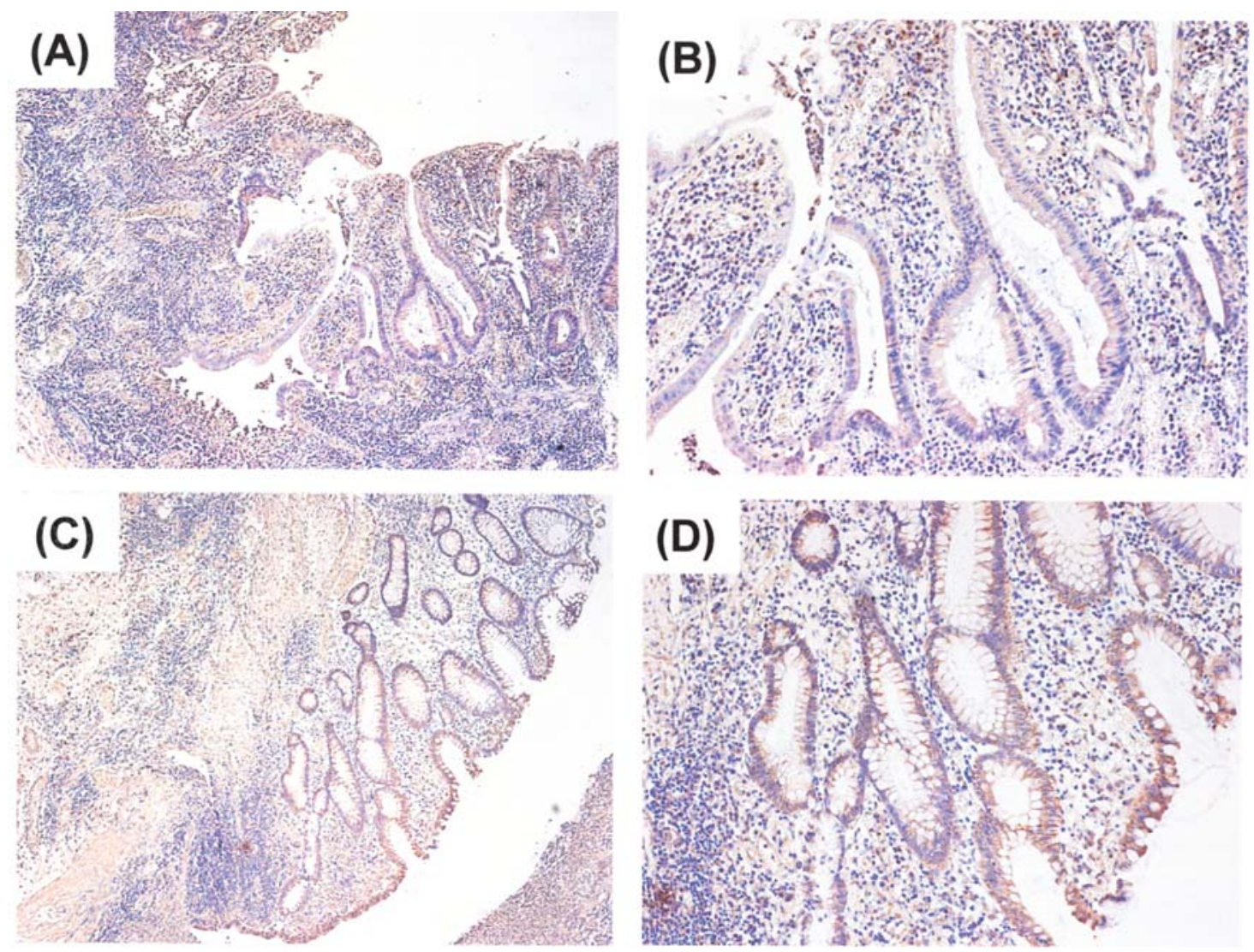

Figure 4. Epiregulin expression in IBD mucosa. (A) and (B), ulcerative colitis; and (C) and (D), Crohn's disease. Original magnification (A) x100. 
inflamed mucosa of IBD patients. Amphiregulin and epiregulin were not expressed in the normal colonic mucosa, but were detectable in the neoplastic lesions and in the IBD mucosa. To our knowledge, this is the first report on the amphiregulin expression in the inflamed mucosa of IBD patients. Furthermore, this is the first immunohistochemical analysis of the epiregulin expression in neoplastic lesions in the colon and IBD mucosa. These observations suggest that amphiregulin and epiregulin play a role in tumor growth and mucosal repair in the colon.

In various cells, amphiregulin and epiregulin are bifunctional growth factors. Recently, we demonstrated that recombinant amphiregulin and epiregulin stimulated the proliferation of human colonic myofibroblasts in a dose- and time-dependent manner (21). Previous studies also showed that amphiregulin and epiregulin stimulate the proliferation of colonic epithelial cells $(11,12,17,22)$. This suggests that amphiregulin and epiregulin play a crucial role in the process of wound healing through their action on both epithelial and mesenchymal cells in the intestine. This hypothesis may be supported by previous reports which indicate that mice deficient in EGFRs show an increased susceptibility to DSSinduced colitis $(17,23)$.

In this study, we found that the amphiregulin and epiregulin expression was increased in epithelial and mesenchymal cells from the IBD mucosa. We have recently reported that IL-1ß and TNF- $\alpha$ induced the amphiregulin and epiregulin expression in colonic myofibroblasts (21). The proinflammatory cytokines IL- $1 \beta$ and TNF- $\alpha$ promote inflammatory responses and regulate aspects of cellular immunity which are important for host defence against infection. The expression of IL-1ß and TNF- $\alpha$ was reported to be elevated in the inflamed mucosa of inflammatory bowel disease. These findings suggest that proliferative responses in inflamed colonic mucosa are mediated by proinflammatory stimuli-induced amphiregulin and epiregulin in both autocrine and paracrine fashions. It is likely that proinflammatory cytokines such as IL- $1 \beta$ and TNF- $\alpha$ may play an important role in the process of tissue remodelling and wound healing through the induction of amphiregulin and epiregulin in the intestinal mucosa. EGF may also coordinate with these proinflammatory cytokines to promote tissue repair processes via the induction of amphiregulin and epiregulin.

Colonic carcinogenesis involves a stepwise accumulation of mutations in tumor-suppressor genes and proto-oncogenes (24). These mutations in turn deregulate the mechanisms controlling crypt cell proliferation, maturation and apoptosis that are normally controlled by multiple homeostatic mechanisms, including signals from the EGFR. Targets of these signalling pathways include cyclin D1 and cyclooxygenase-2 (COX-2). Cyclin D1 is an important EGFR target that controls G1-S cell cycle progression. COX-2 is the prevalent and rate-limiting enzyme required for prostaglandin biosynthesis. In human colon cancers, alterations in ErbB receptors, such as the up-regulation of EGFR and ErbB2 and increases in EGFR ligands including transforming growth TGF- $\alpha$, have been described (24). Increases in the EGFR or ErbB2 expression portend a greater invasiveness of these tumors and a worse prognosis. Our observations of the amphiregulin and epiregulin expression in adenomas and carcinomas indicate that autocrine and paracrine secretion mechanisms of these factors are involved in tumor growth in the colon.

In conclusion, this study demonstrated the amphiregulin and epiregulin expression in neoplastic and inflammatory lesions in the colon. The findings suggest that amphiregulin and epiregulin may play an important role in tumor growth and mucosal repair in the colon.

\section{References}

1. Yarden Y and Sliwkowski MX: Untangling the ErbB signalling network. Nat Rev Mol Cell Biol 2: 127-137, 2001.

2. Chowdhury A, Fukuda R and Fukumoto S: Growth factor mRNA expression in normal colorectal mucosa and in uninvolved mucosa from ulcerative colitis patients. J Gastroenterol 31: 353-360, 1996.

3. Tarnawski AS and Jones MK: The role of epidermal growth factor (EGF) and its receptor in mucosal protection, adaptation to injury, and ulcer healing: involvement of EGF-R signal transduction pathways. J Clin Gastroenterol 27: S12-S20, 1998.

4. Beck PL and Podolsky DK: Growth factors in inflammatory bowel disease. Inflamm Bowel Dis 5: 44-60, 1999.

5. Shoyab M, McDonald VL, Bradley JG and Todaro GJ: Amphiregulin: a bifunctional growth-modulating glycoprotein produced by the phorbol 12-myristate 13-acetate-treated human breast adenocarcinoma cell line MCF-7. Proc Natl Acad Sci USA 85: 6528-6532, 1988.

6. Plowman GD, Green JM, McDonald VL, et al: The amphiregulin gene encodes a novel epidermal growth factor-related protein with tumor-inhibitory activity. Mol Cell Biol 10: 1969-1981, 1990.

7. Castillo J, Erroba E, Perugorria MJ, et al: Amphiregulin contributes to the transformed phenotype of human hepatocellular carcinoma cells. Cancer Res 66: 6129-6138, 2006.

8. Normanno N, Selvam MP, Qi CF, et al: Amphiregulin as an autocrine growth factor for c-Ha-ras- and c-erbB-2-transformed human mammary epithelial cells. Proc Natl Acad Sci USA 91: 2790-2794, 1994.

9. Piepkorn M, Lo C and Plowman G: Amphiregulin-dependent proliferation of cultured human keratinocytes: autocrine growth, the effects of exogenous recombinant cytokine and apparent requirement for heparin-like glycosaminoglycans. J Cell Physiol 159: 114-120, 1994

10. Chu EK, Foley JS, Cheng J, Patel AS, Drazen JM and Tschumperlin DJ: Bronchial epithelial compression regulates epidermal growth factor receptor family ligand expression in an autocrine manner. Am J Respir Cell Mol Biol 32: 373-380, 2005.

11. Johnson GR, Saeki T, Gordon AW, Shoyab M, Salomon DS and Stromberg K: Autocrine action of amphiregulin in a colon carcinoma cell line and immunocytochemical localization of amphiregulin in human colon. J Cell Biol 118: 741-751, 1992.

12. Damstrup L, Kuwada SK, Dempsey PJ, et al: Amphiregulin acts as an autocrine growth factor in two human polarizing colon cancer lines that exhibit domain selective EGF receptor mitogenesis. Br J Cancer 80: 1012-1019, 1999

13. Beales IL: Gastrin and interleukin-1ß stimulate growth factor secretion from cultured rabbit gastric parietal cells. Life Sci 75: 2983-2995, 2004

14. Toyoda H, Komurasaki T, Uchida D, et al: Epiregulin. A novel epidermal growth factor with mitogenic activity for rat primary hepatocytes. J Biol Chem 270: 7495-7500, 1995.

15. Shirakata Y, Komurasaki T, Toyoda H, et al: Epiregulin, a novel member of the epidermal growth factor family, is an autocrine growth factor in normal human keratinocytes. J Biol Chem 275: 5748-5753, 2000.

16. Takahashi M, Hayashi K, Yoshida K, et al: Epiregulin as a major autocrine/paracrine factor released from ERK- and p38MAPK-activated vascular smooth muscle cells. Circulation 108: 2524-2529, 2003.

17. Lee D, Pearsall RS, Das S, Dey SK, Godfrey VL and Threadgill DW: Epiregulin is not essential for the development of intestinal tumors but is required for protection from intestinal damage. Mol Cell Biol 24: 8907-8916, 2004.

18. Rachmilewitz D: Coated mesalazine (5-aminosalicylic acid) vs. sulphasalazine in the treatment of active ulcerative colitis: a randomised trial. BMJ 298: 82-86, 1989. 
19. Best WR, Becktel JM and Singleton JW: Rederived values of the eight coefficients of the Crohn's disease activity index (CDAI). Gastroenterology 77: 843-846, 1979.

20. Fujino S, Andoh A, Bamba S, et al: Increased expression of interleukin-17 in inflammatory bowel disease. Gut 52: 65-70, 2003.

21. Inatomi O, Andoh A, Yagi Y, Bamba S, Tsujikawa T and Fujiyama Y: Regulation of amphiregulin and epiregulin expression in human colonic subepithelial myofibroblasts. Int $\mathbf{J}$ Mol Med 18: 497-503, 2006.

22. Baba I, Shirasawa S, Iwamoto R, et al: Involvement of deregulated epiregulin expression in tumorigenesis in vivo through activated Ki-Ras signalling pathway in human colon cancer cells. Cancer Res 60: 6886-6889, 2000.
23. Egger B, Procaccino F, Lakshmanan J, et al: Mice lacking transforming growth factor $\alpha$ have an increased susceptibility to dextran sulfate-induced colitis. Gastroenterology 113: 825-832, 1997.

24. Fichera A, Little N, Jagadeeswaran S, et al: Epidermal growth factor receptor signalling is required for microadenoma formation in the mouse azoxymethane model of colonic carcinogenesis. Cancer Res 67: 827-835, 2007. 